\title{
Energy density field approach for low- and medium-frequency Vibroacoustic analysis of a car body using a probabilistic computational model
}

\author{
Laurent Gagliardini, Morad Kassem \\ PSA-Peugeot-Citroën- France \\ Christian Soize \\ Université Paris-Est, France
}

Copyright $\odot 2008$ SAE International

\begin{abstract}
In this paper, a new energy-density field approach is proposed for low- medium-frequency vibroacoustic analysis of complex industrial structures, using a probabilistic computational model. The observed structure is composed of a trimmed body coupled and its internal cavity. The objective of this paper is to take advantage of some statistical properties of the frequency response functions to build a simplified vibroacoustic model. In this approach, the Frequency Response Functions (FRF) of the vibroacoustic system are expressed as the product of a dimensionless "smooth" matrix and local mobilities or impedances, depending on their type (acoustical, vibratory or vibroacoustic). The stochastic computational model of the vibroacoustic system is obtained from the reduced mean computational model and is using a nonparametric probabilistic approach. Thus, both the data uncertainties and the model uncertainties are taken into account. The stochastic equations are solved using the Monte Carlo method providing independent realizations of the dimensionless FRF of the vibroacoustic system. The confidence regions around the mean value of both the conventional FRFs and the dimensionless ones are estimated using quantiles. The mean frequency response functions are used to compute the principal local axes regarding the structural local sensitivities (direction of maximum mobility). Both types of FRFs are then projected over these principal axes. This projection provides useful information about archetypal characteristics of structural components and avoids local correlation problems. Above a selected frequency, some interesting spectral and spatial properties of the dimensionless frequency response functions appear; smooth variations allow building the expected simplified model, by gathering redundant information in the excitation and observation areas.
\end{abstract}

\section{INTRODUCTION}

Vibroacoustic design of automotive vehicles is becoming a more and more complex task due to the increasing demand of acoustic comfort. Thus, car makers are forced to increase the quality of the design process while keeping its cost within affordable limits. However, in the current design process, the complexity of an automotive vehicle structure as well as the numerous noise sources lead to consider a very large number of Frequency Response Functions (FRF), making the analysis a very exhausting process. Moreover, the existence of modeling uncertainties (data and model errors) as well as product uncertainties increases the difficulty of analyses. In a context of development costs reduction, the need for more robust and simplified models becomes a necessity.

In the past years, statistical methods such as the Statistical Energy Analysis (SEA) [1] and its derivations were adopted by the automotive industry as a powerful tool for the analysis of vibroacoustic behavior of complex structures in the high-frequency range. These methods are very efficient because they synthesize the dynamical behavior of a structure in a few robust quantities such as exchanged power or energies. Unfortunately, they do not apply in the low- and medium-frequency ranges, due to some specific assumptions or pre-requisites such as space and frequency homogeneity of responses.

Recently developed probabilistic vibroacoustic models now allow investigating the ensemble statistical properties of the dynamic responses of any industrial product (including design diversity as well as manufacturing uncertainties). In the present work an energy density field approach based on a probabilistic computational model is proposed. The use of a 
probabilistic model is essential since it allows a statistical analysis even when space or frequency responses statistics are not relevant. This permits the application of energy approaches in the low- and medium- frequency ranges and hopefully the rise of interesting properties.

In the first part of the paper, the general structuralacoustic problem of a vehicle at low frequencies is presented, along with current modeling tools. Then, the application of the nonparametric probabilistic approach is introduced, leading to the construction of independent realization of the system dynamic responses (FRF). In a third part, a novel energy density field approach is proposed. The method requires a projection on specific local coordinates, which are introduced in a fourth part. Finally, numerical results are presented, showing immediate benefits of the proposed energy method on an automotive vehicle case.

\section{VIBROACOUSTIC PROBLEM ANALYSIS}

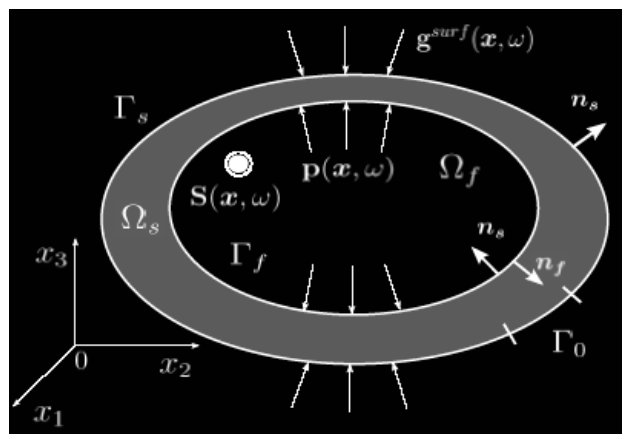

Figure1: Vibroacoustic system modeling

The vibroacoustic problem of an automotive vehicle is modeled by a structure coupled with its internal acoustic cavity [2]. Surface or volume forces are applied to the structure and acoustic sources are placed inside the internal acoustic cavity. Such a system is shown in Fig.1. The analysis of the system consists in studying the FRFs between various excitation and observation points. For any structural problem, for example, the linear dynamic response may be written:

$$
\mathbf{V}=\mathbf{T F}
$$

where $\mathbf{V}$ is the vector of the velocity response of the structure, and $\mathbf{F}$ is the vector of the excitation forces.

The previous relation can be extended to the more general case of random excitation as:

$$
\mathbf{s}^{\mathbf{v}}=\mathbf{T} \mathbf{s}^{\mathbf{f}} \mathbf{T}^{+}
$$

where $\mathbf{s}^{\mathbf{v}}$ and $\mathbf{s}^{\mathbf{f}}$ are respectively the cross-spectral matrices of the response velocity and of the excitation force.
The velocity FRF matrix, $\mathbf{T}$, is calculated by inverting the dynamical stiffness matrix, $\mathbf{A}$, obtained from a finite element modeling:

$$
\mathbf{T}=j \omega \mathbf{A}^{-1}
$$

Where $\mathbf{A}=-\omega^{2} \mathbf{M}+\mathbf{K}+j \omega \mathbf{D}$, and $\mathbf{M}, \mathbf{K}, \mathbf{D}$ are respectively the mass, stiffness and damping matrices.

Due to the large number of degrees of freedom in automotive vehicles finite elements models a model reduction is needed to reduce the simulation time. This model reduction is obtained by projecting the vibroacoustic problem equations on the elastic modes of the structure and the acoustic modes of the internal cavity to obtain the following equation:

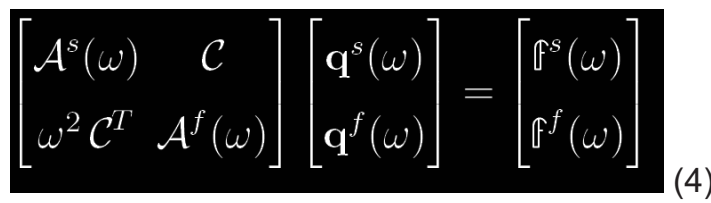

Where $A^{s}$ and $A^{f}$ are respectively the reduced dynamical stiffness matrices of the structure and the acoustic cavity. $\mathrm{C}$ is the coupling matrix. $\mathbf{q}^{\boldsymbol{s}}$ and $\mathbf{q}^{f}$ are the generalized coordinates. $\mathbb{F}^{s}$ and $\mathbb{F}^{f}$ are the generalized force and acoustic flow rate vectors. The relation between the physical and the generalized coordinates can then be written as follows

$$
\mathbf{u}^{s}(\omega)=\Psi \mathbf{q}^{s}(\omega) \quad, \quad \mathbf{p}^{f}(\omega)=\Phi \mathbf{q}^{f}(\omega)
$$

where $\Psi$ (resp. $\Phi$ ) are the elastic structural modes (resp. the acoustic modes), and $\mathbf{u}^{s}$ and $\mathbf{p}^{f}$ are the displacement and pressure vectors of the response in the physical coordinates.

This approach is very efficient for the calculation of the FRF for an automotive vehicle structure; it is implemented in most of commercial numerical simulation tools.

\section{ENSEMBLE SIMULATION USING THE NONPARAMETRIC PROBABILISTIC APPROACH}

The application of the nonparametric probabilistic approach is explained briefly in this section and the reader is referred to references [3][4] for the general formulation and [5] for an application in the automotive industry.

The nonparametric probabilistic approach consists in replacing the matrices of the reduced mean vibroacoustic computational model by random matrices whose probability distribution is explicitly constructed using the available information i.e. the deterministic 
matrices (matrices of the mean reduced model). One then obtains from Eq.4 the following random equation

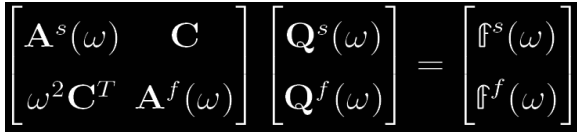

where $\mathbf{Q}^{\mathbf{S}}$ (resp. $\mathbf{Q}^{f}$ ) are the random generalized coordinates of the structure (resp. of the acoustic cavity).

The random matrices can be presented in an algebraic form $[3,4]$. This allows independent realizations of the Monte Carlo method to be obtained. Fixed dispersion parameters are used to construct random matrices with a given variance. A random variable generator is used to ensure the independency of the obtained realizations. The reduced random dynamic stiffness matrix is then inverted for each realization of the random reduced matrices, providing a realization of the generalized coordinates. The results are then projected on the physical degrees of freedom and the FRF's between chosen excitations and observation points are extracted.

In this work the number of modes, as well as the number of independent realization necessary to achieve the convergence of the stochastic simulation was taken from reference [5] which used a similar model.

After convergence, the statistics of the results such as the mean value of the realizations can easily be calculated. The confidence regions around the mean values of the FRF are constructed using the quantiles method [6] as explained in references [3] and [4].

\section{NOVEL ENERGY DENSITY FIELD APPROACH}

A new energy density field approach is proposed in this section. The method is derived as explained in reference [7] with one major limitation: the cross-spectral density function of the input forces has to be a diagonal matrix.

In the proposed approach, the usual FRF relation between inputs and outputs is replaced by a power transfer equation

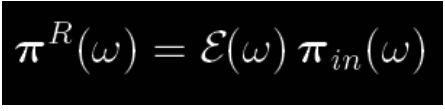

where the power input is related to the external forces by:

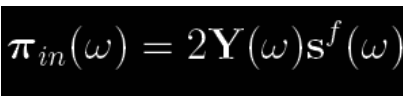

and the dimensionless frequency response matrix $\mathcal{E}(\omega)$ appears to be:

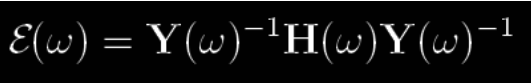

where $\mathbf{H}$ is the term to term square matrix of the FRF matrix and $\mathbf{Y}$ is the input point mobility matrix such as

$$
\mathbf{Y}(\omega)=\operatorname{Re}\left\{\frac{\mathbf{V}}{\mathbf{F}}\right\}
$$

The response power, $\pi^{R}(\omega)$, is related to the velocity spectral density, by:

$$
\mathbf{s}^{v}(\omega)=\mathbf{Y}(\omega) \boldsymbol{\pi}^{R}(\omega)
$$

The proposed approach is practically interesting when the mobility matrix is diagonal. Omitting the off-diagonal terms would result in loss of information. Thus, to prevent this loss of information the FRF are projected into adapted local coordinates defined by the principal directions of local mobilities at one point or at several points. This coordinates system, as well as the projection of the FRFs, are shown in the next section.

\section{ADAPTED LOCAL COORDINATES SYSTEM DEFINED BY THE PRINCIPAL DIRECTIONS OF THE MEAN MOBILITIES}

The local coordinates system chosen for the analysis is obtained from the mean mobility values at one point ( 3 or 6 degrees of freedom) or several points ( $\mathrm{N}$ degrees of freedom). In this paper, only the single point with 3 DOFs case will be discussed. The local coordinates system is obtained by solving the eigenvalue problem of the $3 \times 3$ mobility matrix averaged over all the Monte Carlo realizations, such as :

$$
\mathbb{T}_{p}(\omega)=\mathbb{X}_{p}(\omega) \Lambda(\omega) \mathbb{K}_{p}(\omega)^{T}
$$

where

$$
\mathbb{T}_{p}(\omega)=\Re e\left\{E\left\{\mathbf{T}_{p}(\omega)\right\}\right\}
$$
is the

mean mobility matrix of point $p$

$p$ is the basis of the local coordinates, mobilities.

$\Lambda$ is the diagonal matrix of the principal

It can be seen in (10) that the local coordinate system, as well as the principal mobilities are functions of frequency. After the eigenvalue problem is solved for each frequency, and every studied point, each realization of the FRF is projected on the local basis to obtain the random local FRF matrix

$$
\mathbf{T}_{p}^{l o c}(\omega)=\mathbb{X}_{p}(\omega)^{T} \mathbf{T}_{p}(\omega) \mathbb{X}_{p}(\omega)
$$

After assembly over all the DOF of the system, the equations of the energy density field approach can be obtained in the local coordinates and the equation of the 
dimensionless frequency response matrix can be rewritten in the local coordinates

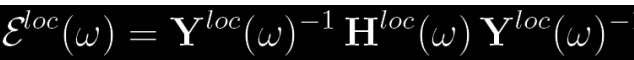

This approach can be extended to the 6 DOFs case at one point, or more DOFs involving several points.

\section{NUMERICAL RESULTS}

The energy density field approach was applied to a numerical model of a production car. The finite element model of the numerical model structure and its internal cavity is shown in Fig. 2.

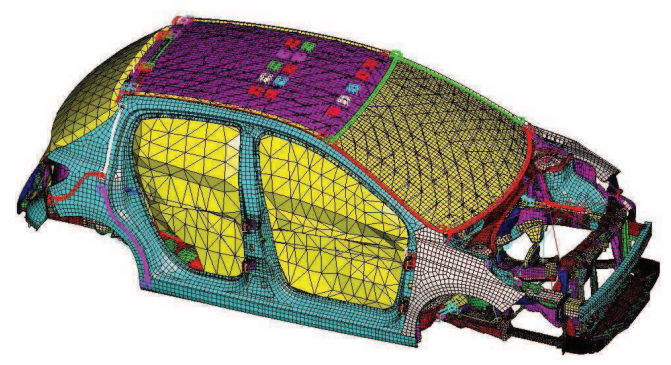

Figure 2: Finite element model of the vehicle structure and its internal acoustic cavity

The model consists of 1042851 DOF for the structure, 9157 DOF for the acoustic cavity. The frequency band of analysis was chosen to be $B=[50,350] \mathrm{Hz}$. Only translational DOF were observed for the structure. In the frequency band of analysis the structure has 1958 modes including the 6 rigid body modes and the acoustic cavity has 160 modes including the constant pressure mode. Forces were applied to twenty eight points of the structure including the powertrain mounts and the front suspension connections, while twelve acoustic sources were placed in the acoustic cavity for a total of 96 DOF. Observation points were assigned to each excitation point in order to obtain a square FRF matrix.

\section{FREQUENCY RESPONSE ANALYSIS}

Six hundred realizations are necessary for the convergence of the Monte Carlo method for both the structure and the acoustic cavity. Fig. 3 shows the mean values of the Monte Carlo simulation of the usual FRF and of the dimensionless FRF. Results are presented for a force excitation in the three local directions at one point of the structure and observation at one structural DOF of a distant point.

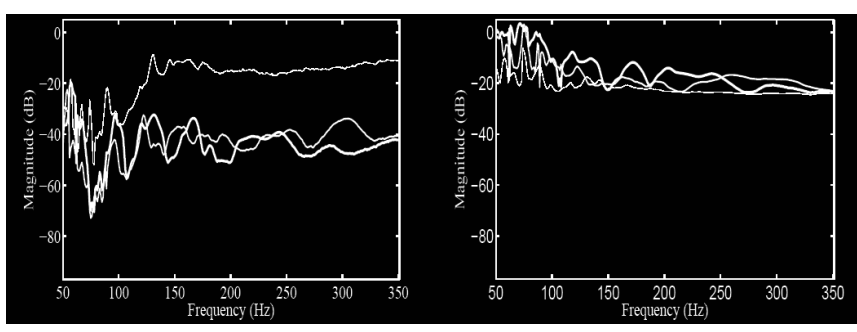

Figure 3 Mean values of the usual FRF (left figure) and dimensionless FRF (right figure). Excitation: at 3 structural DOF of the right engine mount, observation: maximum mobility direction on the roof.

It can be seen that the dimensionless FRF is smoother than the usual FRF. Moreover, when exciting in the three local DOF, the resulting dimensionless FRF's have very close values and tend to converge towards an asymptotic value, contrary to the usual FRF's. This shows that, above a given frequency, these dimensionless FRF are slightly dependent of the loading direction. Reciprocally, they are also nearly independent of the observation direction. This property could lead to a significant reduction of the number of FRF necessary to control the car body design.

In addition, the dimensionless FRF's have been shown to be much less sensitive to uncertainties than the usual FRF's as shown in Fig.4. The proposed formulation is thus more robust regarding the model and parameters uncertainties.
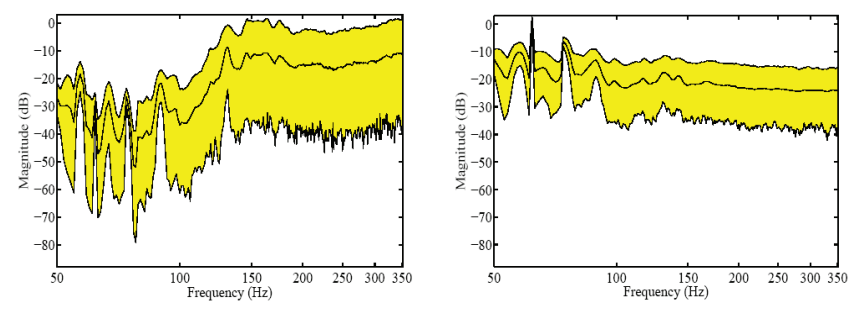

Figure 4: Confidence regions of the dimensional FRF (left) and dimensionless FRF (right figure). Excitation: right engine mount in its maximum mobility direction, observation: single point on the roof in its maximum mobility direction.

Considering expression (6) in the view of the observed results, one may say that the vibration transfer between two points may be separated into local effects described by the diagonal mobility matrices, and a global robust energy density transfer effect characterized by the dimensionless frequency response matrix.

Similar results are observed for acoustic and vibroacoustic transfer functions (see [4]).

\section{LOCAL COORDINATE SYSTEM ANALYSIS}

The values of the mean mobilities in the three local principal directions are shown in Fig. 5 for the roof and fig. 6 for the right engine mount.

For a thin structure such as the roof, one direction shows a mobility 1000 times higher than other directions. This direction logically appears to be close to the geometrical 
normal of the roof at the considered point. This result shows the ability of the local mobility analysis to separate flexural effects from membrane effects.

For the right engine mount, located on the right longitudinal front member of the car body, 2 directions present similar mobilities, while the third is about ten times lower. This is typical of a beam-like component.

In addition to the previous benefits associated to the proposed energy approach, valuable information on the archetypal behavior of the structure is provided by the local coordinate analysis.

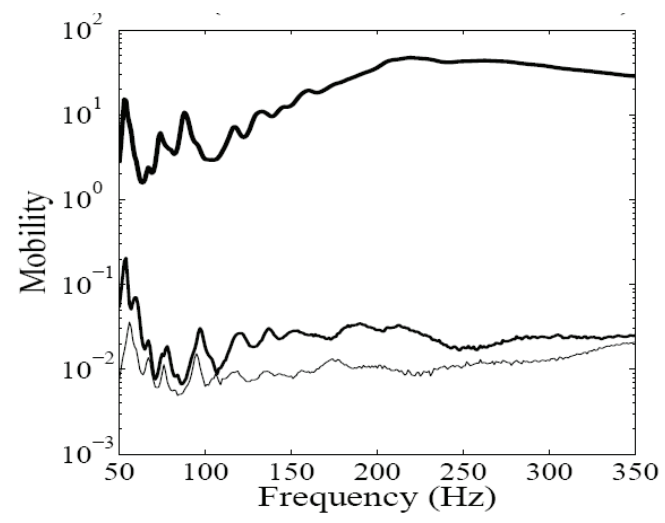

Figure 5: 3 principal values of mobility versus frequency for a point located on the roof

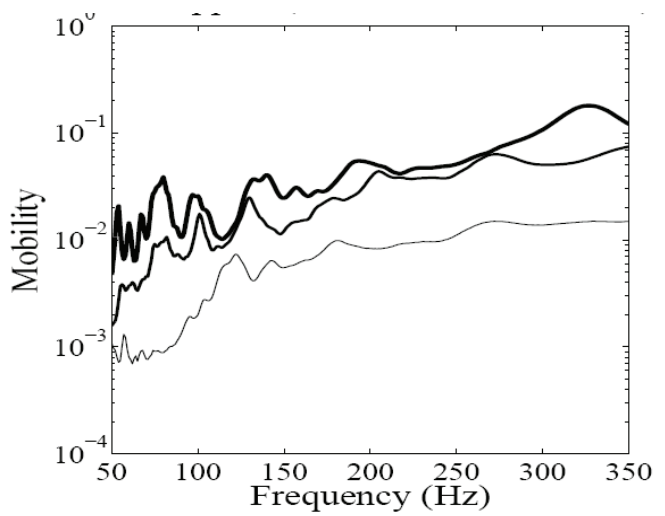

Figure 6: 3 principal values of mobility versus frequency at the right engine mount location

\section{CONCLUSION}

An energy density field approach based on a probabilistic computational model was presented. This approach addresses the complexity of the industrial noise problems in several ways.

First, it simplifies the expression of a system vibroacoustic response by using an energy parameter that appears to be mostly independent of the direction of excitation or the direction of observation. Thus, the number of FRF of the system could be considerably decreased. In such a way, it separates global effects described by the dimensionless transfer matrix- from local effects -described by principal values of mobility-. Second, it simplifies the expression of the excitation sources which are characterized by means of input power. Finally, this approach uses the nonparametric probabilistic approach of model and data uncertainties, providing probabilistic responses. Results does not only concern one single object but an ensemble including product and process uncertainties as well as modeling uncertainties. This approach is robust regarding these uncertainties, since appropriate values of the probabilistic model parameters are used.

Based on the above mentioned properties, a simplification -substructuring- of the dimensionless matrix seems possible by gathering degrees of freedom having similar behavior and therefore providing another simplification of the model. This is the next step of this work in progress.

\section{REFERENCES}

1. R.H. Lyon, Statistical Energy Analysis of Dynamical Systems, MIT Press, San Diego, 1975.

2. Ohayon, R. and Soize, C. (1998). Structural Acoustics and Vibration, Mechanical Models Variational Formulations and Discretization

3. C. Soize, A comprehensive overview of a nonparametric probabilistic approach of model uncertainties for predictive models in structural dynamics, Journal of Sound and Vibration, 288, pp. 623-652, 2005.

4. C. Soize, Random matrix theory for modeling uncertainties in computational mechanics. Comput. Methods Appl. Mech. Engrg., 194, pp. 1333-1366, 2005.

5. Durand, J.-F., Gagliardini, L. and Soize, C. (2008). "Structural-acoustic modeling of automotive vehicles in presence of uncertainties and experimental identification and validation", Journal of the Acoustical Society of America 24, 1513-1525.

6. R.J. Serfling, Approximation Theorems of mathematical Statistics, Wiley,New york, 1980.

7. M. Kassem, C. Soize, L. Gagliardini, Energy density field approach for low- and medium-frequency vibroacoustic analysis of complex structures using a statistical computational model. The Journal of Sound and Vibration, accepted for publication, 2008.

8. L. Gagliardini, G. Borello, Virtual SEA: Towards an industrial process, SAE International, 2007-01-2302, 2007.

\section{CONTACT}

laurent.gagliardini@mpsa.com 